\title{
Experimental realization of dynamo action: present status and prospects
}

\author{
André Giesecke ${ }^{1}$, Frank Stefani ${ }^{1}$, Thomas Gundrum ${ }^{1}$, \\ Gunter Gerbeth $^{1}$, Caroline Nore ${ }^{2}$ and Jacques Léorat ${ }^{3}$ \\ ${ }^{1}$ Helmholtz-Zentrum Dresden-Rossendorf \\ P.O.B. 510119, D-01314, Dresden, Germany \\ email: a.giesecke@hzdr.de \\ ${ }^{2}$ Laboratoire d'Informatique pour la Mécanique et les Sciences de l'Ingénieur (LIMSI), CNRS, \\ BP 133, F-91403 Orsay cedex, France \\ email: nore@limsi.fr \\ ${ }^{3}$ Observatoire de Paris-Meudon, \\ place Janssen, F-92195 Meudon, France \\ email: jacques.leorat@obspm.fr
}

\begin{abstract}
In the last decades, the experimental study of dynamo action has made great progress. However, after the dynamo experiments in Karlsruhe and Riga, the von-KármánSodium (VKS) dynamo is only the third facility that has been able to demonstrate fluid flow driven self-generation of magnetic fields in a laboratory experiment. Further progress in the experimental examination of dynamo action is expected from the planned precession driven dynamo experiment that will be designed in the framework of the liquid sodium facility DRESDYN (DREsden Sodium facility for DYNamo and thermohydraulic studies).

In this paper, we briefly present numerical models of the VKS dynamo that demonstrate the close relation between the axisymmetric field observed in that experiment and the soft iron material used for the flow driving impellers. We further show recent results of preparatory water experiments and design studies related to the precession dynamo and delineate the scientific prospects for the final set-up.
\end{abstract}

Keywords. magnetic fields, methods: laboratory, methods: numerical

\section{Introduction}

Liquid metal dynamo experiments present a complementary tool to gain further insight into the working principles of astrophysical magnetic field generation, for example by allowing a verification of scaling laws obtained from numerical simulations or by providing measurements in a level of detail that cannot be reached with astronomical observations. Looking at the typical laboratory scale $(\mathcal{L} \sim 1 \mathrm{~m})$, the fluid flow driven generation of magnetic fields is a demanding task that requires typical flow velocities of the order of $10 \mathrm{~m} / \mathrm{s}$ in order to cross the dynamo threshold at all. So far, only three facilities have been able to demonstrate fluid flow driven self-generation of magnetic fields (Stefani et al., 2008). The first confirmation of fluid flow driven dynamo action under laboratory conditions occurred nearly simultaneously at two distinct experiments conducted in Riga (Gailitis et al., 2000) and in Karlsruhe (Stieglitz \& Müller, 2001). The Karlsruhe dynamo experiment essentially consisted of a cylindrical assembly of helical guiding tubes thus roughly mimicking the (assumed) flow in the Earth's liquid core. The small scale helical structure of the flow configuration was suitable for applying a two-scale separation and the observed generation of a large scale magnetic field could be well described using mean-field theory. 
An explanation of induction action from a mean flow field was also appropriate for the Riga dynamo (Gailitis et al., 2004). The principle of this experiment is based on a flow configuration proposed by Ponomarenko (1973), who showed that an infinite helical flow embedded in a stationary conductor can show dynamo action at a rather low magnetic Reynolds number. The realization in the experiment is carried out in a tall cylinder with a single propeller driving a flow along the axial direction. In contrast to the Karlsruhe dynamo which has been deconstructed a couple of years ago, the Riga dynamo is still operating allowing further investigations like, e.g., a transition to chaotic behavior that has recently been found numerically by Stefani et al. (2011).

\section{The von-Kármán-Sodium dynamo}

In the VKS dynamo experiment a flow of liquid sodium is driven by two counterrotating impellers that are located close to the end-caps of a cylindrical vessel (see left panel in figure 1). Dynamo action was found at a surprising low magnetic Reynolds number of $\mathrm{Rm} \approx 32$. In dependence of the flow driving various regimes could be explored which show different dynamical properties, like bursts, oscillations or sudden field reversals (Monchaux et al., 2007; Berhanu et al., 2007). A striking property of the VKS dynamo is the exclusive occurrence of (mainly axisymmetric) dynamo action only in cases when the flow is driven by impellers that are made of a soft iron alloy with a relative permeability of $\mu_{\mathrm{r}} \approx 65$ (Monchaux et al., 2009; Verhille et al., 2010).
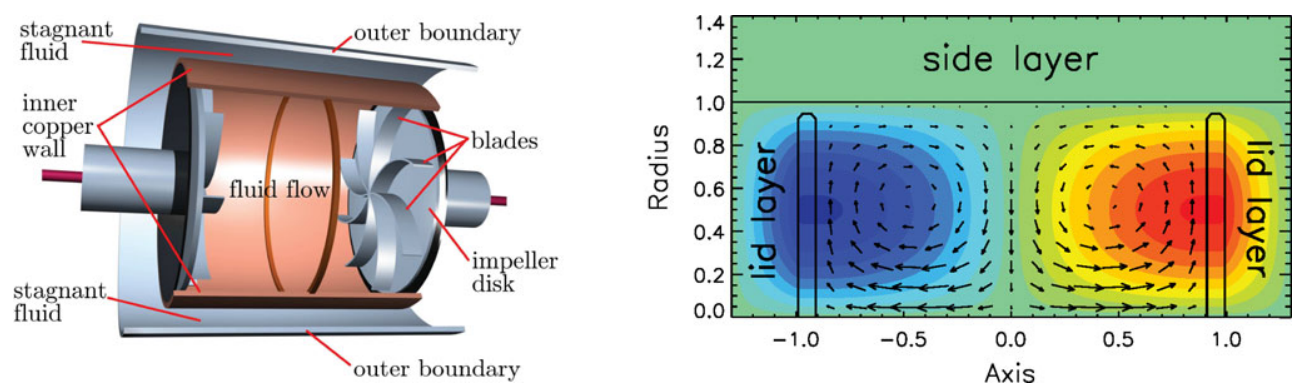

Figure 1. Left: Sketch of the original setup of the VKS dynamo. Later, the inner copper walls have been removed so that the flow active regime was enlarged and the aspect ratio (height over diameter) decreased. Right: Mean velocity field applied in the numerical simulations. The color coded structure presents the azimuthal velocity and the arrows denote the meridional velocity contribution $\left(v_{r}, v_{z}\right)$. The velocity field stems from analytical expressions specified by Marié et al. (2006). Two disk-like sub-domains with radius $R_{\mathrm{imp}}=0.95$ are located in the intervals $z \in[-1.0 ;-0.9]$ and $z \in[0.9 ; 1.0]$ and represent soft iron disks of thickness $d=0.1$.

In order to include the impact of a non-uniform distribution of the relative permeability $\mu_{\mathrm{r}}$ the induction equation must be written in the form

$$
\frac{\partial \boldsymbol{B}}{\partial t}=\nabla \times\left(\boldsymbol{u} \times \boldsymbol{B}+\frac{1}{\mu_{\mathrm{r}} \mu_{0} \sigma} \frac{\nabla \mu_{\mathrm{r}}}{\mu_{\mathrm{r}}} \times \boldsymbol{B}-\frac{1}{\mu_{\mathrm{r}} \mu_{0} \sigma} \nabla \times \boldsymbol{B}\right),
$$

where $\boldsymbol{u}$ is the prescribed (mean) flow, $\boldsymbol{B}$ the magnetic flux density, $\mu_{0}$ the vacuum permeability $\left(\mu_{0}=4 \pi \cdot 10^{-7} \mathrm{Vs} / \mathrm{Am}\right)$, and $\sigma$ the electrical conductivity. We have performed numerical simulations of (2.1) using a prescribed analytical flow field which resembles the average flow resulting from a von-Kármán-like forcing (the so called MND-flow, see right panel of figure 1 and Marié et al., 2006). The soft iron material of the impeller disks is modelled by two axisymmetric permeability distributions. The results in terms of eigenmodes and related growth-rates reveal a close connection between the exclusive 
occurrence of dynamo action in the presence of soft-iron impellers and the observed axisymmetry of the magnetic field (see figure 2 and Giesecke et al., 2012).
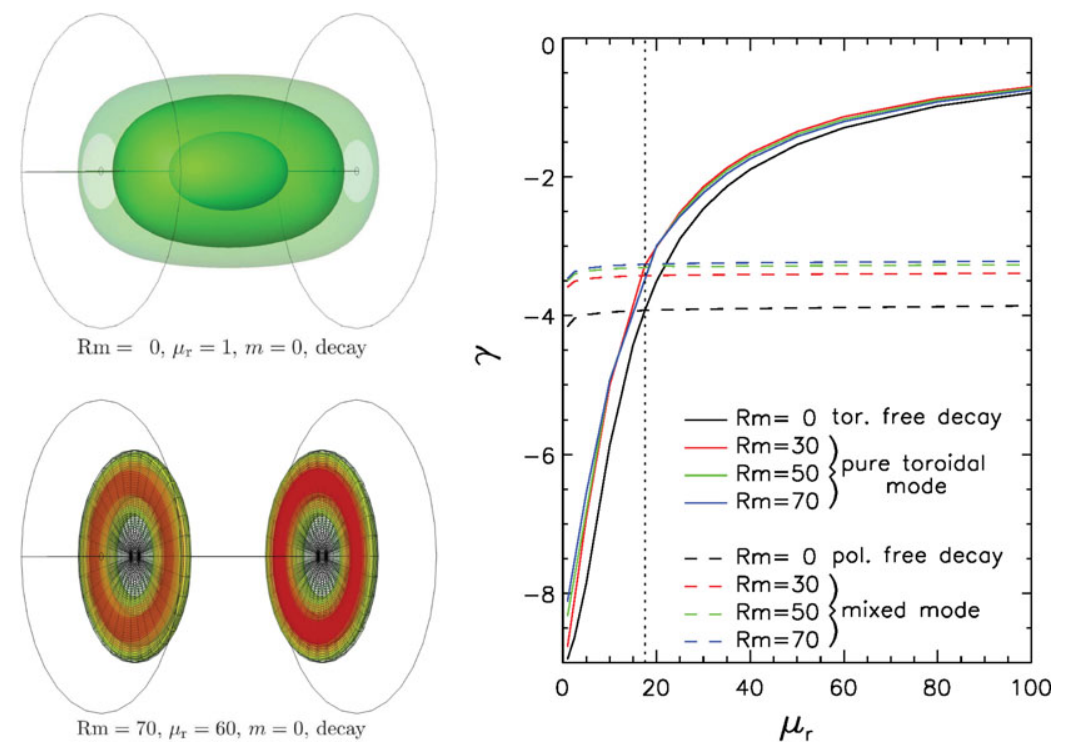

Figure 2. Field structure and growth-rates for the axisymmetric eigenmodes. The iso-surfaces on the left present the magnetic energy density of the $m=0$ mode for various sets of the control parameters $\mathrm{Rm}$ and $\mu_{\mathrm{r}}$. The plot on the right hand side shows the corresponding growth-rates of the $m=0$ eigenmode (solid curves: purely toroidal mode, dashed curve: mixed mode).

Here we consider only the behavior of the axisymmetric eigenmodes, for which we find two distinct classes of eigenmodes. A so called mixed mode, consisting of a poloidal and a toroidal contribution, is largely independent of the permeability. This mode always decays on a rather fast timescale. Furthermore, we observe a purely toroidal mode that is considerably enhanced with increasing $\mu_{\mathrm{r}}$. This mode essentially stems from the paramagnetic pumping generated at the interface between fluid and soft-iron disk (Giesecke et al., 2012) and is largely independent of the flow magnitude. Increasing the disk permeability this mode is shifted close to the dynamo threshold and becomes the leading eigenmode of the system within the experimentally relevant regime (i.e. for $\mathrm{Rm} \leqslant 50$ and $\mu_{\mathrm{r}} \approx 65$ ). However, regarding the utilized axisymmetric numerical setup the purely toroidal mode is not able to become a growing eigenmode due to the restrictions resulting from Cowling's theorem. Hence, a satisfying explanation of the observed axisymmetric dynamo mode requires mean field effects like the $\alpha$-effect. Since the flow is considerably turbulent such effects are undoubtedly operative, however, so far their properties (e.g. spatial distribution or amplitude) are only speculative. The $\alpha$-effect is closely related to the kinetic helicity via the well know relation $\alpha \sim \tau / 3\langle\boldsymbol{u} \cdot(\nabla \times \boldsymbol{u})\rangle$ (Krause \& Rädler, 1980). Very recently, Ravelet et al. (2012) presented a rough estimate for the kinetic helicity $h_{\text {kin }}=\langle\boldsymbol{u} \cdot(\nabla \times \boldsymbol{u})\rangle$ obtained from numerical simulations which shows strong spatial concentrations close to the impellers. The corresponding magnitude for $\alpha$ is definitely within the regime that was required in Giesecke et al. (2010) to allow for growing axisymmetric dynamo modes (using a uniform $\alpha$-distribution). 


\section{A precession driven dynamo}

The DREsden Sodium facility for DYNamo and thermohydraulic studies (DRESDYN) is a scheduled infrastructure project which will serve as a platform for large-scale experiments related to geo-and astrophysics as well as for thermohydraulic experiments on liquid metal applications in energy related technologies (Stefani et al., 2012). The most elaborate facility in the framework of DRESDYN will be a precession-driven dynamo experiment. Further planned experiments are a large Taylor-Couette type experiment for the combined investigation of the magneto-rotational instability and the Tayler instability and various small scale experiments related to the thermo-hydraulics of liquid sodium. In the following, we will give a brief description of the setup of the precession dynamo and a short summary of preliminary results from a preparatory water experiment.

\subsection{Motivation and theoretical background}

Precession has often been regarded as an alternative or complementary driving mechanism for the geodynamo in order to overcome inconsistencies related to the age of the Earth's solid inner core (Malkus, 1968). The precession of the Earth's axis represents a significant variance of an orbital parameter which undoubtedly influences the Earth's inner core flow and hence the geodynamo. This is supported by modulations of the inter-reversal time distribution with a period $\tau \sim 100 \mathrm{kyrs}$ which is rather close to the Milankovich cycle period that describes an oscillation in the eccentricity of the Earth's orbit (Consolini \& De Michelis, 2003). A dynamo experiment with a fluid flow driven by precession is further attractive because a conducting fluid simultaneously rotating around two axis provides the preconditions for ideal homogeneous dynamo action without any internal guiding tubes (as in Karlsruhe) or propellers (as in Riga and the VKS dynamo).

In a co-rotating system subject to precessional driving the flow is determined by the Navier-Stokes equation including source terms for the Coriolis- and the Poincaré forces:

$$
\frac{\partial}{\partial t} \boldsymbol{u}+(\boldsymbol{u} \nabla) \boldsymbol{u}+2(\boldsymbol{\omega}+\boldsymbol{\Omega}(\boldsymbol{t})) \times \boldsymbol{u}=\nu \nabla^{2} \boldsymbol{u}-\nabla \Phi-(\boldsymbol{\Omega}(\boldsymbol{t}) \times \boldsymbol{\omega}) \times \boldsymbol{r} .
$$

In eq. (3.1) $\boldsymbol{u}$ is the velocity field, $\boldsymbol{\omega}$ denotes the angular velocity of the fluid container, $\boldsymbol{\Omega}$ is a time-dependent vector that describes the precession of $\boldsymbol{\omega}$ and $\Phi$ is the reduced pressure (including the centrifugal term). Numerical simulations in various geometries have shown that a flow described by (3.1) indeed is able to drive a dynamo (sphere, Tilgner, 2005; cylinder, Nore et al., 2011; cube, Krauze, 2010; spheroid, Wu \& Roberts, 2009). However, so far the experimental verification which of course will be realized at parameters that will be quite different from the numerical simulations is missing and reliable conclusions for natural dynamos remain difficult.

\subsection{Experimental set-up}

The precession dynamo scheduled in the framework of DRESDYN will consist of a cylindrical container with approximately $2 \mathrm{~m}$ diameter, rotating with up to $\omega_{\text {cyl }}=10 \mathrm{~Hz}$ around its axis. The vessel will additionally rotate with up to $\omega_{\mathrm{p}}=1 \mathrm{~Hz}$ around a second axis, the precession axis, whose angle with respect to the first axis can be varied between $90^{\circ}$ and $45^{\circ}$ (see right panel in figure 3 for a preliminary sketch). In preparation for the liquid sodium experiment and in order to determine the optimal geometric configuration as well as essential process parameters a smaller water experiment has been developped (see left panel in figure 3). This small scale experiment is similar to the ATER experiment (Léorat, 2006; Mouhali, 2010) but is equipped with additional sensors that provide the determination of torques and motor powers needed to drive the rotation of the cylinder and the turntable. Further problems that are attacked in the water experiment are the 

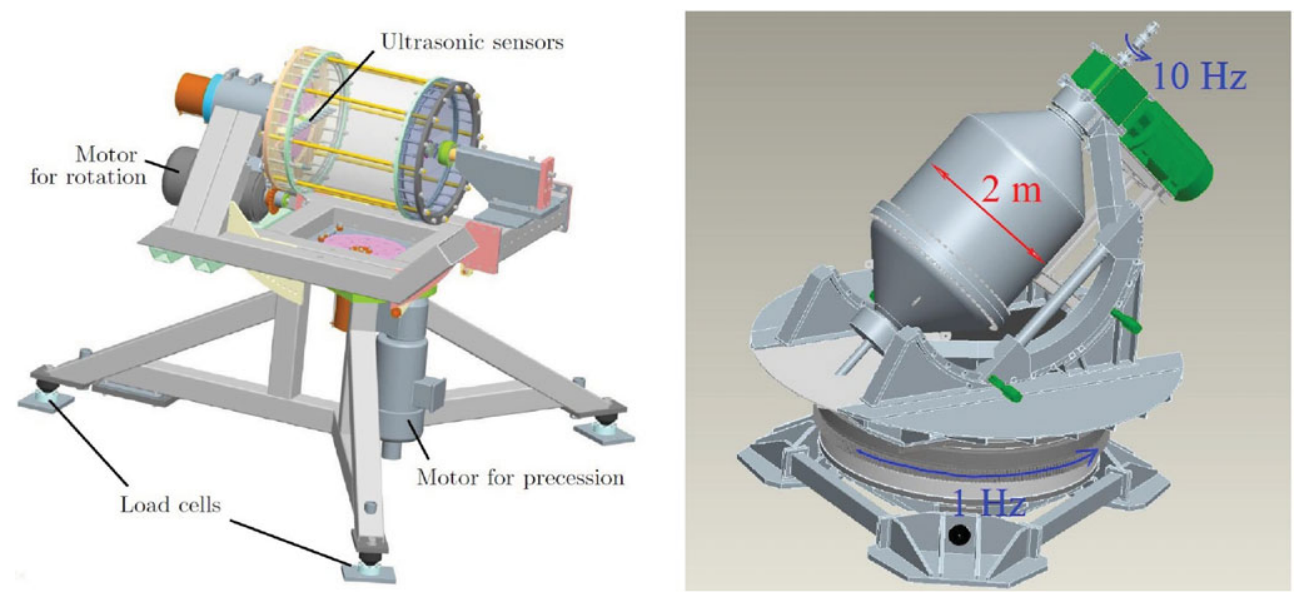

Figure 3. Left: Sketch of the small-scale water precession experiment (1:6 downscaled). The experiment provides the possibility to observe the velocity field (via Ultrasonic Doppler Velocimetry (UDV)). Right: Draft sketch for the large-scale precession dynamo experiment. The frequencies denote the maximum achievable frequencies for rotation and precession. The diameter of the inner cylinder will be approximately $2 \mathrm{~m}$ with an aspect ratio close to 1 .

estimation of the gyroscopic torques acting on the basement and the estimation of the average flow that can be applied for kinematic simulations.

Up to present, various ultrasonic devices are installed on the end caps of the cylinder allowing the determination of the axial velocity component using Ultrasonic Doppler Velocimetry (UDV). In the slowly rotating regime and for low precession rates $\Gamma=$ $\omega_{\mathrm{p}} / \omega_{\text {cyl }}$ we first observe a laminar flow comprising only a few non-axisymmetric modes. The large scale flow component is determined by an azimuthal wavenumber $m=1$ and the flow structure is typical for a Kelvin mode which is fixed in the frame of the turntable (see figure 4). For $\omega_{\text {cyl }}=0.2 \mathrm{~Hz}$ the typical velocity amplitudes in the co-rotating system
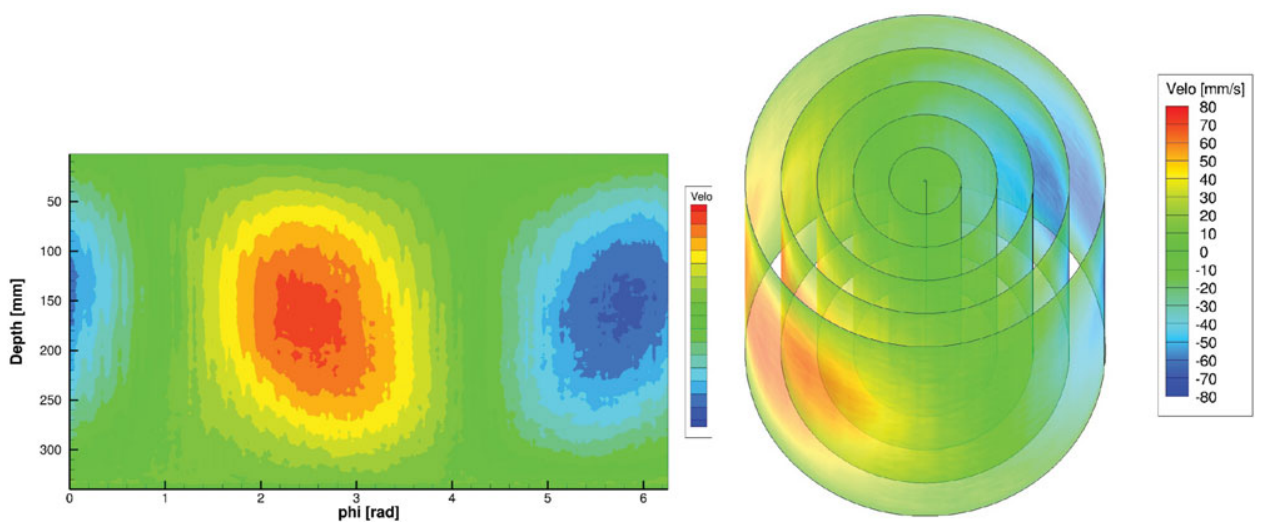

Figure 4. Pattern for a weakly precessing flow $\left(\omega_{\text {cyl }}=0.2 \mathrm{~Hz}\right.$ and $\left.\omega_{\mathrm{p}}=0.01 \mathrm{~Hz}\right)$. The colors denote the axial velocity $u_{z}$ in the co-rotating frame. A clear non-axisymmetric mode with an azimuthal wavenumber $m=1$ has emerged. The maximum velocity magnitude is roughly one third of the rotation of the container.

(i.e. the container-wall frame) are of the order of $u_{z} \approx 40 \mathrm{~mm} / \mathrm{s}$. Scaled to the 6 times larger liquid sodium facility and to a rotation rate of $\omega_{\text {cyl }}=10 \mathrm{~Hz}$ this would result in a a value of $12 \mathrm{~m} / \mathrm{s}$ which correponds to a magnetic Reynolds number of $\mathrm{Rm} \sim 120$. 
Increasing the precession rate above $\Gamma \approx 0.07$ the flow abruptly switches into a fully turbulent state which is accompied by a sharp increase of the required motor power. So far, the fully turbulent regime (as well as the transitional regime) cannot be reached in numerical simulations. However, from the water experiment it is already obvious that the flow properties in both regimes are different with the simple $m=1$ mode being suppressed in the turbulent state so that we also expect surprising effects for dynamo action, e.g. an increased impact of the magnetic field on the fluid flow.

\section{Conclusions}

Fluid flow driven laboratory dynamo action is an established phenomenon but still not easy to achieve. Further progress is expected utilizing "natural" flows as energy source for a dynamo as it is planned for the precession dynamo experiment in the framework of DRESDYN. For the slow rotation rate examined so far the flow structure most probably is too simplistic to provide for dynamo action. Nevertheless, the preparatory water experiments show that precessional flow driving is quite efficient and will allow to reach magnetic Reynolds numbers that will be in the range of the critical value required to achieve precessional driven dynamo action in a sphere (Tilgner, 2005). More complex flow geometries are expected for higher precession rates and the next goal for the flow measurements is an identification of helical eddies that have been found by Mouhali (2010) at the ATER experiment.

\section{References}

Berhanu, M. et al. 2007, Europhys. Lett., 77, 59001

Consolini, G. \& De Michelis, P. 2003, Phys. Rev. Lett., 90, 058501

Gailitis, A. et al. 2000, Phys. Rev. Lett., 84 (19), 4365-4368

Gailitis, A. et al. 2004, Phys. Plasmas, 11, 2838-2843

Giesecke, A., Stefani, F. \& Gerbeth, G. 2010, Phys. Rev. Lett., 104, 044503

Giesecke, A. et al. 2012, New J. Phys., 14 (5), 053005

Krauze, A. 2010, Magnetohydrodynamics, 46 (3), 271-280

Krause, F. \& Rädler, K.-H., Mean-field magnetohydrodynamics and dynamo theory, Oxford, Pergamon Press, 1980

Léorat, J. 2006, Magnetohydrodynamics, 42 (2-3), 143-151

Malkus, W. V. R.. 1968, Science, 160, 259-264

Marié, L., Normand, C. \& Daviaud, F. 2006, Phys. Fluids, 18, 017102

Monchaux, R. et al. 2007, Phys. Rev. Lett., 98, 044502

Monchaux, R. et al. 2009, Phys. Fluids, 21 (3), 035108

Mouhali, W. 2010, PhD Thesis, Université Paris-Diderot - Paris VII

Nore, C., Léorat, J., Guermond, J.-L. \& Luddens, F. 2011, Phys. Rev. E, 84 (1), 016317

Stefani, F., Gailitis, A., \& Gerbeth, G. 2008, Z. Angew. Math. Mech., 88, 930-954

Stefani, F., Gailitis, A., \& Gerbeth, G. 2011, Astron. Nachr., 332, 4

Stefani, F. et al. 2012, Magnetohydrodynamics, 48 (1), 103-113

Stieglitz, R. \& Müller, U. 2001, Phys. Fluids, 13, 561-564

Tilgner, A. 2005, Phys. Fluids, 17 (3), 034104

Ponomarenko, Y.-B. 1973, J. App. Mech. Tech. Phys., 14, 775-778

Ravelet, F., Dubrulle, B., Daviaud, F. \& Ratié, P.-A. 2012, Phys. Rev. Lett., 109 (2), 024503

Verhille, G. et al. 2010, New J. Phys., 12 (3), 033006

Wu, C.-C \& Roberts, P. 2009, Geophys. Astrophys. Fluid Dyn., 103 (6), 467-501 